\title{
Mensuração de Poder de Mercado por Meio de Dados de Marcas Comerciais: Modelos, Limitações e Aplicações
}

\author{
Measurement of Market Power Using Trademark Data: \\ models, limitations and applications
}

DANILO R. D. AGUIAR *,*

\begin{abstract}
RESUMO: Este artigo analisa os principais modelos utilizados para medir o poder de mercado por meio de dados da marca e verifica as vantagens do modelo desenvolvido por Cotterill, Franklin e Ma sobre os demais. Este é um modelo muito flexível que pode ser usado para quaisquer suposições conjeturais e permite a medição do poder de mercado unilateral e colusivo. Várias formas de aplicação são sugeridas e perspectivas muito promissoras são reconhecidas para esse tipo de estudo.
\end{abstract}

PALAVRAS-CHAVE: Poder de mercado; competição; estimação de elasticidades.

ABSTRACT: This paper analyzes the main models used to measure market power by means of brand data and verifies the advantages of the model developed by Cotterill, Franklin and $\mathrm{Ma}$ over the others. This is a very flexible model which can be used to any conjectural assumptions and allows the measurement of both unilateral and collusive market power. Several ways of application are suggested, and very promising perspectives are recognized for this type of study.

KEYWORDS: Market power; competition; elasticity estimation.

JEL Classification: D43; L13; L25.

\section{INTRODUÇÃO}

Poder de mercado pode ser definido como a habilidade das firmas em manterem seus preços acima do custo marginal, com vistas na obtenção de lucro econô-

\footnotetext{
* Professor adjunto da Universidade Federal de Viçosa, Viçosa/MG, Brasil. E-mail: danilo@ufv.br.

* O O autor é grato ao CNPq pelo financiamento do programa de pós-doutorado em que este trabalho foi iniciado. É grato, também, ao prof. John Connor, da Purdue University (EUA), pelas discussões mantidas sobre o tema em apreço.
} 
mico. De acordo com a fonte de origem, o poder de mercado pode ser classificado em dois tipos: poder cooperativo (ou colusivo) e poder unilateral.

O poder cooperativo origina-se do processo em que as firmas agem coordenadamente para aumentar o lucro conjunto, seja por meio da redução da produção, seja pelo aumento do preço. Num contexto de cooperação total, as firmas teriam o grau máximo possível de poder de mercado, e a indústria teria um desempenho semelhante ao do monopólio. A cooperação pode ser explícita, como nos cartéis (proibidos pelas leis antitruste da maioria dos países), ou implícita, quando as firmas sinalizam umas para outras, tentando mostrar suas ações na expectativa de que as outras ajam coerentemente, para aumentar o lucro conjunto. O oposto da cooperação é a rivalidade, caracterizada por firmas agindo umas contra as outras; por exemplo, se a firma A reduz sua produção (ou aumenta seu preço), as demais passam a produzir mais (ou a cobrar preços menores), com o intuito de conquistar parte da parcela de mercado da firma A. ${ }^{1}$

O poder de mercado unilateral, como o próprio nome sugere, não depende das ações e das reações das firmas concorrentes. Diz-se que uma firma tem poder unilateral quando existem poucos substitutos para seu(s) produto(s) e/ou quando os compradores acreditam que os substitutos existentes não são suficientemente bons. Dessa forma, o poder unilateral decorre da existência de barreiras à entrada no mercado (ou segmento de mercado) e da diferenciação do produto. As barreiras são elementos que desencorajam ou impedem o ingresso no mercado, tais como vantagens de custo para as firmas estabelecidas, dificuldade de acesso a insumos essenciais, barreiras legais (patentes) etc. A diferenciação do produto, por sua vez, decorre da melhoria dos atributos do produto ou de propaganda. Um dos extremos do poder de mercado unilateral ocorre quando o produto é totalmente diferenciado, e a ausência de substitutos faz desta indústria um monopólio. Alternativamente, se não houver nenhum poder de mercado unilateral, a indústria tende a funcionar como um mercado contestável (Baumol, 1982), havendo eficiência máxima. A propaganda, além de tornar a demanda mais inelástica, por convencer os consumidores da não-existência de substitutos potenciais, aumenta o poder de mercado, por incrementar o custo de entrada no mercado. Isto porque o ingressante potencial teria de despender muito em promoção para conquistar parcela viável em mercados em que existem marcas consolidadas.

Muito embora se reconhecesse a existência dos dois diferentes tipos de poder de mercado, apenas recentemente tem sido possível medi-los, visto que é recente a disponibilidade de séries de preços e quantidades de marcas comerciais comercializadas. Essas séries são instrumentos de fundamental importância para a mensuração do poder de mercado, porque a concorrência se dá, em mercados de produtos diferenciados, entre marcas comerciais, e não entre firmas. É nesse nível de

\footnotetext{
${ }^{1}$ Ambos os tipos de comportamento, cooperação e rivalidade, têm sido incorporados aos modelos tradicionais de oligopólio por meio das variações conjeturais (do inglês conjectural variations). Ver Martin (1993).
} 
desagregação que as estimações precisam ser feitas para se inferir o tipo de poder de mercado que predomina. Mas que estimações seriam essas? Grosso modo, são estimadas elasticidades da demanda que permitem mostrar como os consumidores reagem a cada marca e como as marcas interagem entre si.

Vários modelos têm sido propostos, e grande número de estimações tem sido feito sobre demandas de marcas comerciais. Alguns dos modelos têm assumido conjeturas específicas, tal como Nash-Bertrand, em que as firmas supõem que os preços de suas concorrentes não se alteram quando elas mudam seus próprios preços; outros modelos têm permitido um teste ex-post do grau de rivalidade ou cooperação entre as marcas. Este artigo propõe-se a analisar os modelos que vêm sendo usados e detalhar, em especial, o modelo desenvolvido por Cotterill, Franklin \& Ma (1996), doravante referidos como CFM, no tocante a suas limitações e aplicações. A apresentação dos modelos teóricos é feita no item 2; o modelo empírico usado por CFM é apresentado no item 3; o item 4 discute possíveis aplicações do método; e o item 5 conclui o trabalho.

\section{ASPECTOS TEÓRICOS}

Os primeiros modelos para estimação da demanda de produtos diferenciados a partir de dados de marcas comerciais foram desenvolvidos por Baker \& Breshnahan (1985 e 1988), Hausman, Leonard \& Zona (1994); e Cotterill \& Haller (1997). Baker \& Breshnahan estimaram demandas residuais, enquanto Hausman et al. e Cotterill \& Haller assumiram conjetura tipo Nash-Bertrand e estimaram sistemas de demanda unilateral. Ambos os sistemas, o residual e o unilateral, apresentam deficiências dignas de menção . O sistema de demanda residual apresenta hipóteses muito restritivas (com o intuito de simplificar a estimação) e não permite a separação dos poderes de mercado unilateral e cooperativo. Num sistema de $\mathrm{n}$ marcas, Baker \& Breshnahan removem os efeitos de preços e quantidades das marcas nas quais eles não estão interessados, estimando demandas residuais apenas para as marcas de interesse. O sistema de demanda unilateral, por outro lado, só considera o poder unilateral, já que, por assumir comportamento tipo Nash-Bertrand, supõe nulo o poder cooperativo. Além disso, uma conjetura tipo NashBertrand é claramente irrealista, visto que o preço é a variável mais comumente alterada por uma empresa diante de perda de parcela de mercado.

Mais recentemente, CFM desenvolveram um modelo que apresenta uma série de vantagens sobre seus predecessores. Em relação ao sistema de demanda unilateral, de Hausman et al. e Cotterill \& Haller, a vantagem do modelo de CFM é sua flexibilidade, uma vez que este pode ser utilizado para quaisquer conjeturas de reação das demais marcas. Em vez de requerer a pressuposição de conjeturas específicas antes da estimação, o procedimento de CFM permite um teste ex-post do comportamento estratégico em vigor na indústria. Por isso, admite a existência (e apresenta uma medida) do poder cooperativo, além do unilateral. A vantagem em relação ao sistema de demanda residual, de Baker \& Breshnahan (1985 e 1988), é 
que o modelo de CFM permite a separação dos poderes unilateral e cooperativo, e não exige que as conjeturas sejam consistentes. O problema é que a hipótese de consistência (que significa que a conjetura feita sobre a reação de preços por parte dos concorrentes teria de ocorrer na prática), implicitamente assumida na formulação de Baker \& Breshnahan, não se aplica a muitos dos modelos de oligopólio existentes. Além dessas vantagens, CFM mostraram que tanto o sistema de demanda unilateral quanto o residual eram casos especiais do modelo mais geral por eles desenvolvido.

De maneira mais detalhada, CFM assumiram preço como variável estratégica, gerando a seguinte demanda para a marca comercial 1:

$$
\mathrm{q}_{1}=\mathrm{q}_{1}\left(\mathrm{p}_{1}, \ldots, \mathrm{p}_{\mathrm{n}}, \underline{\mathrm{D}}\right),
$$

em que $\mathrm{q}_{1}=$ quantidade da marca $1 ; \mathrm{p}_{1}=$ preço da marca $\mathrm{i}(\mathrm{i}=1, \ldots, \mathrm{n}) ; \underline{\mathrm{D}}=$ vetor das outras variáveis modificadoras da demanda.

Derivando a equação (1) em relação a $\mathrm{p}_{1}$ (regra da cadeia) e fazendo algumas manipulações algébricas, chega-se a uma fórmula que envolve apenas elasticidades:

$$
\eta_{1}^{0}=\eta_{11}+\sum_{i=2}^{n}\left(\eta_{1 i} \cdot \varepsilon_{i 1}\right)
$$

em que

$\eta_{1}^{0}=$ elasticidade-preço observável da demanda da marca 1 , baseada em condições reais de mercado (e não em conjeturas);

$\eta_{11}=$ elasticidade-preço parcial da demanda da marca 1 , sem levar em consideração mudanças em preços de outras marcas;

$\eta_{1 ;}=$ elasticidade-preço cruzada da demanda da marca 1 , em relação aos preços das outras marcas $\left(\mathrm{p}_{\mathrm{i}}\right)$;

$\gamma_{1}=$ elasticidade de reação de preços das marcas rivais de 1 (porcentagem de mudança em $p_{i}$, quando $p_{1}$ varia $1 \%$ ).

A título de interpretação, a equação (2) pode ser divida em duas partes. A primeiram fzm, é uma medida do poder de mercado unilateral, uma vez que essa elasticidade não leva em conta as reações das outras marcas. A segunda parte, correspondente ao somatório de $\eta_{\mathrm{li}} \gamma_{\mathrm{il}}$, é uma medida do poder de mercado cooperativo. Três casos especiais merecem ser mencionados a respeito da equação (2). O primeiro é o caso em que as firmas não reagem a uma variação de preço da marca 1 , conhecido como caso Nash-Bertrand, em que $\gamma_{\mathrm{il}}=0$ e $\eta_{1}^{0}=\eta_{\mathrm{li}}$. Isto implica poder de mercado cooperativo nulo. O segundo caso, denominado colusivo, é caracterizado por $\gamma_{\text {il }}$ positivo (assim como $\eta_{\mathrm{li}}$, para marcas comerciais substitutas entre si), implicando demanda observável mais inelástica do que a unilateral. Quanto maior for o grau de colusão entre as marcas, mais inelástica será a demanda da marca 1 . O terceiro caso a ser mencionado, denominado rivalidade, caracteriza-se por $\gamma_{\text {il }}$ negativo, resultando na redução de poder de mercado, pelas ações das rivais da marca 1 (a demanda observável é mais elástica do que a unilateral).

Embora as elasticidades apresentadas na equação (2) já sejam, como se mos- 
trou acima, medidas dos poderes de mercado unilateral e cooperativo, existe ainda a necessidade de padrões que permitam melhor interpretação dos parâmetros estimados. CFM desenvolveram esses padrões seguindo a lógica da teoria da organização industrial, ou seja, mantendo competição perfeita e monopólio como situações extremas. Os índices de poder de mercado propostos por CFM podem ser ilustrados na Figura 1, que mostra três curvas de demanda que estão relacionadas com as elasticidades apresentadas na equação (2). A demanda totalmente colusiva é a mais inclinada; a unilateral é a menos inclinada; e a demanda observável fica entre as duas últimas. ${ }^{2}$ No caso de um aumento do preço da marca representada na Figura 1, a quantidade vendida diminuiria, claramente, menos para colusão total do que para colusão parcial (demanda observável) ou ausência de colusão (demanda unilateral).

Figura 1:Relações Teóricas da Demanda para uma Marca num Oligopólio Diferenciado

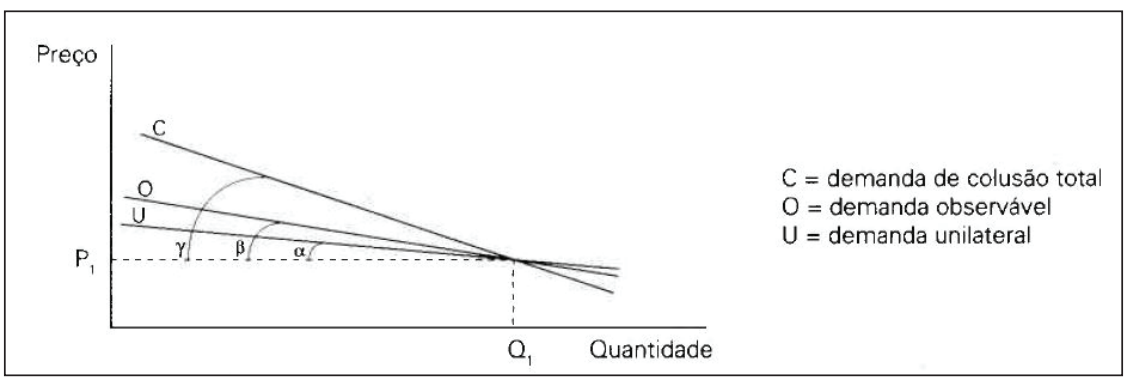

Fonte: adaptado do Cotterill, Franklin \& Ma (1996).

Um padrão para o poder de mercado unilateral já existe e está amplamente difundido nos livros-texto de organização industrial: o índice de Rothschild $(\mathrm{R})^{3}$. Este índice é representado pela inclinação da demanda unilateral (tangente de a), dividida pela inclinação da demanda de colusão total (tangente de y). $\mathrm{Na}$ vizinhança do preço $\mathrm{P}_{1}$ da Figura $1, \mathrm{R}$ pode ser calculado pela divisão da elasticidade-preço de colusão total pela elasticidade-preço unilateral, da seguinte forma:

$$
\mathrm{R}=\frac{\operatorname{tg} \alpha}{\operatorname{tg} \beta}=\frac{\left(\frac{\Delta \mathrm{P}}{\Delta \mathrm{Q}}\right)^{\mathrm{u}}}{\left(\frac{\Delta \mathrm{P}}{\Delta \mathrm{Q}}\right)^{\mathrm{c}}}=\frac{\eta_{1}^{\mathrm{c}}}{\eta_{11}}
$$

Analisando-se a equação (3), verifica-se que $\mathrm{R}$ varia de zero a um, respectiva-

\footnotetext{
${ }^{2}$ A Figura 1 representa uma situação em que algum nível de cooperação existe. No caso de rivalidade, a curva de demanda observável seria menos inclinada do que a unilateral.

${ }^{3}$ Ver Greer (1984, p. 99).
} 
mente para perfeita competição, em que a demanda unilateral seria horizontal, e colusão total, em que a demanda unilateral teria a mesma inclinação da totalmente colusiva e o poder seria o mesmo do monopólio.

CFM propuseram outro índice para medir o poder de mercado observável (ou total, já que combina ambos os tipos de poder). $\mathrm{O}$ índice proposto, denominado índice de Cotterill (C), é obtido pela divisão da inclinação da demanda observável (tangente de B) pela inclinação da demanda de colusão total (tangente de y), ou, alternativamente, pela divisão da elasticidade-preço de colusão total pela elasticidade-preço da demanda observável, como segue:

$$
\mathrm{C}=\frac{\operatorname{tg} \beta}{\operatorname{tg} \gamma}=\frac{\left(\frac{\Delta \mathrm{P}}{\Delta \mathrm{Q}}\right)^{\circ}}{\left(\frac{\Delta \mathrm{P}}{\Delta \mathrm{Q}}\right)^{\mathrm{c}}}=\frac{\eta_{1}^{\mathrm{c}}}{\eta_{1}^{\mathrm{o}}}
$$

Similarmente a $\mathrm{R}$, o índice $\mathrm{C}$ varia de zero a um. Se $\mathrm{C}$ for igual a 1, o mercado estará se comportando monopolisticamente (em razão de cooperação e/ou de diferenciação do produto), e, se $\mathrm{C}$ for igual a zero, existem duas possibilidades: ou não existe poder de mercado ou o poder de mercado unilateral está sendo plenamente compensado por rivalidade entre as marcas.

CFM desenvolveram também uma medida para o poder de mercado cooperativo, denominada quociente de Chamberlin $(\mathrm{CH})$, que é a parcela do poder de mercado observável que não decorre do poder unilateral:

$$
\mathrm{CH}=\frac{\mathrm{C}-\mathrm{R}}{\mathrm{C}}=1-\frac{\eta_{1}^{\circ}}{\eta_{11}}
$$

Se não houver poder de mercado cooperativo, a elasticidade-preço da demanda observável será idêntica à elasticidade-preço da demanda unilateral, o que resulta em $\mathrm{CH}$ igual a zero. $\mathrm{Na}$ presença de algum grau de colusão entre as marcas, o quociente $\mathrm{CH}$ variaria de zero a um, atingindo a unidade no caso de colusão total, em que a elasticidade-preço da demanda observável se aproximaria de zero. $\mathrm{Na}$ presença de rivalidade, a elasticidade-preço observável seria mais elástica do que a elasticidade unilateral, resultando em $\mathrm{CH}$ negativo. Consequentemente, o quociente $\mathrm{CH}$ varia de menos infinito a um.

A implementação empírica do modelo de CFM consiste em estimar as elasticidades unilateral, observável e totalmente colusiva e usar estas elasticidades para calcular os índices de Rothschild e Cotterill e o quociente de Chamberlin para cada marca comercial. A título de exemplo, imagine uma situação fictícia em que existem duas marcas ( 1 e 2 ) e as elasticidades estimadas são ${ }^{4}$ :

$$
\begin{array}{llll}
\eta_{1}^{\mathrm{c}}=-1 ; & \eta_{11}=-2 ; & \eta_{12}=0,5 ; & \varepsilon_{21}=1 ; \mathrm{e} \\
\eta_{2}^{\mathrm{c}}=-0,6 ; & \eta_{22}=-1 ; & \eta_{21}=0,8 ; & \varepsilon_{12}=0,5 .
\end{array}
$$

\footnotetext{
${ }^{4} \mathrm{~A}$ forma de estimação destas elasticidades é apresentada no próximo item.
} 
As elasticidades observáveis são calculadas pela fórmula 2:

$$
\begin{aligned}
& \eta_{1}^{\circ}=-2+(0,5 \times 1)=-1,5 ; \\
& \eta_{2}^{\circ}=-1+(0,8 \times 0,5)=-0,6 .
\end{aligned}
$$

Utilizando-se estes valores e as fórmulas (3), (4) e (5), tem-se:

$$
\begin{aligned}
\mathrm{R}_{1} & =\frac{\eta_{1}^{\mathrm{c}}}{\eta_{11}}=\frac{-1}{-2}=0,5 \\
\mathrm{R}_{2} & =\frac{\eta_{2}^{\mathrm{c}}}{\eta_{22}}=\frac{-0,6}{-1}=0,6 \\
\mathrm{C}_{1} & =\frac{\eta_{1}^{\mathrm{c}}}{\eta_{1}^{\circ}}=\frac{-1}{-1,5}=0,66 \\
\mathrm{C}_{2} & =\frac{\eta_{2}^{\mathrm{c}}}{\eta_{2}^{\circ}}=\frac{-0,6}{-0,6}=1 \\
\mathrm{CH}_{1} & =1-\frac{\eta_{1}^{\circ}}{\eta_{11}}=1-\frac{-1,5}{-2}=0,25 \\
\mathrm{CH}_{2} & =1-\frac{\eta_{2}^{\circ}}{\eta_{22}}=1-\frac{-0,6}{-1}=0,4
\end{aligned}
$$

Os resultados do índice $\mathrm{R}$ mostram que as marcas 1 e 2 têm poder decorrente da diferenciação de produto da ordem de, respectivamente, $50 \%$ e $60 \%$ do poder que teriam, caso as duas marcas fossem gerenciadas conjuntamente (monopólio). Os índices $\mathrm{CH}$ mostram que a coo peração entre as marcas permite-lhes ter poder de determinação de preços equivalente a $25 \%$ e $40 \%$ do que teriam em colusão total, respectivamente para as marcas 1 e 2 . Combinando os dois efeitos, os índices $\mathrm{CH}$ mostram que as duas marcas desfrutam de elevado grau de poder de mercado, e que a marca 2 tem tanto poder quanto um monopolista.

\section{IMPLEMENTAÇÃO EMPÍRICA DO MODELO DE CFM}

Para obter os valores das elasticidades no modelo de CFM, dois tipos de equações são estimados inicialmente - demandas e equações de reações de preços das marcas comerciais. Os parâmetros das equações de demanda são usados para calcular as elasticidades $\eta_{\mathrm{jj}}$, e $\eta_{\mathrm{j} 1}$,. As equações de reações de preços geram os parâmetros, $\mathrm{E}$; , em que j e i representam quaisquer marcas. As elasticidades-preço observáveis são calculadas por meio da equação (2), e as elasticidades de colusão total são calculadas a partir de um procedimento que será apresentado mais adian- 
te. Dessa forma, o requerimento mínimo ${ }^{5}$ de dados necessários para as estimações diz respeito às séries temporais dos preços e das quantidades comercializadas de cada marca comercial. CFM estimaram demandas por meio do Almost Ideal Demand System (AIOS), desenvolvido por Deaton \& Muellbauer (1980). Este modelo pode ser representado por:

$$
w_{i}=\alpha_{i}+\sum_{i} \gamma_{i j} \ln p_{i}+\beta_{i} \ln \left(\frac{x}{P}\right)
$$

em que $w_{1}$ é a parcela da marca $i$ na despesa; $p_{i}$ é o preço da marca $j ; x$ é a despesa total com o grupo de marcas; e $P$ é o índice de preços do AIDS ou o índice de preços de Stone, gerando, neste último caso, o modelo denominado Linear Approximate Almost Ideal Demand System ( LA/AIDS). ${ }^{6}$

As equações de reações de preços são deduzidas pela substituição das equações do AIDS nas funções de lucro ${ }^{7}$ Empiricamente, para $n$ marcas, $n$ equações de reação de preços são estimadas, cada uma com um dos preços como variável dependente, e os demais, como explicativas:

$$
\begin{aligned}
& \ln p_{1}=\theta_{1}+\varepsilon_{12} \ln p_{2}+\varepsilon_{13} \ln p_{3}+\ldots+\varepsilon_{1 n} \ln p_{n}, \\
& \ln p_{2}=\theta_{2}+\varepsilon_{21} \ln p_{1}+\varepsilon_{23} \ln p_{3}+\ldots+\varepsilon_{2 n} \ln p_{n}, \\
& \ln p_{n}=\theta_{n}+\varepsilon_{n 1} \ln p_{1}+\varepsilon_{n 2} \ln p_{2}+\ldots+\varepsilon_{n(n-1)} \ln p_{n-1} .
\end{aligned}
$$

A elasticidades de reações de preços são os parâmetros yl], estimados em (7). Dessa forma, as equações (6) e (7) são estimadas conjuntamente, para o cálculo das elasticidades-preço observáveis da demanda, das elasticidades-preço cruzadas da demanda e das elasticidades de reações de preços.

As elasticidades cuja forma de estimação ainda não foi explicitada são as totalmente colusivas. Para explicar como elasticidades do tipo totalmente colusivo poderiam ser calculadas, CFM utilizaram, por simplificação, um sistema com três marcas comerciais, sendo as três marcas gerenciadas pela mesma firma e os preços escolhidos de forma a maximizar o lucro conjunto. A função de lucro é:

$$
\pi=\mathrm{p}_{1} \mathrm{q}_{1}+\mathrm{p}_{2} \mathrm{q}_{2}+\mathrm{p}_{3} \mathrm{q}_{3}-\mathrm{c}\left(\mathrm{q}_{1}, \mathrm{q}_{2}, \mathrm{q}_{3},\right),
$$

em que $p_{i}$ e $q_{i}$ são, respectivamente, o preço e a quantidade da marca i ( $i=1,2,3$ ).

Diferenciando a expressão acima em relação aos preços, a condição de primeira ordem fica:

\footnotetext{
${ }^{5}$ Este é o requerimento mínimo, porque outras variáveis de controle são muitas vezes necessárias para melhorar o ajuste ou para identificar as equações.

${ }^{6}$ Ver Deaton \& Muellbauer (1980).

${ }^{7}$ Ver CFM e Ma (1997).
} 


$$
\begin{aligned}
& \frac{\partial \pi}{\partial p_{1}}=q_{1}+p_{1} \frac{\partial q_{1}}{\partial p_{1}}+p_{2} \frac{\partial q_{2}}{\partial p_{1}}+p_{3} \frac{\partial q_{3}}{\partial p_{1}}-\frac{\partial c}{\partial q_{1}} \frac{\partial q_{1}}{\partial p_{1}}-\frac{\partial c}{\partial q_{2}} \frac{\partial q_{2}}{\partial p_{1}}-\frac{\partial c}{\partial q_{3}} \frac{\partial q_{3}}{\partial p_{1}}=0 \\
& \frac{\partial \pi}{\partial p_{2}}=q_{2}+p_{1} \frac{\partial q_{1}}{\partial p_{2}}+p_{2} \frac{\partial q_{2}}{\partial p_{2}}+p_{3} \frac{\partial q_{3}}{\partial p_{2}}-\frac{\partial c}{\partial q_{1}} \frac{\partial q_{1}}{\partial p_{2}}-\frac{\partial c}{\partial q_{2}} \frac{\partial q_{2}}{\partial p_{2}}-\frac{\partial c}{\partial q_{3}} \frac{\partial q_{3}}{\partial p_{2}}=0 \\
& \frac{\partial \pi}{\partial p_{3}}=q_{2}+p_{1} \frac{\partial q_{1}}{\partial p_{3}}+p_{2} \frac{\partial q_{2}}{\partial p_{3}}+p_{3} \frac{\partial q_{3}}{\partial p_{3}}-\frac{\partial c}{\partial q_{1}} \frac{\partial q_{1}}{\partial p_{3}}-\frac{\partial c}{\partial q_{2}} \frac{\partial q_{2}}{\partial p_{3}}-\frac{\partial c}{\partial q_{3}} \frac{\partial q_{3}}{\partial p_{3}}=0
\end{aligned}
$$

O sistema (8) pode ser simplificado para:

$$
\begin{aligned}
& w_{1}+w_{1} \eta_{11} p c m_{1}+w_{2} \eta_{21} p c m_{2}+w_{3} \eta_{31} p c m_{3}=0 ; \\
& w_{1}+w_{1} \eta_{12} p c m_{1}+w_{2} \eta_{22} p c m_{2}+w_{3} \eta_{32} p c m_{3}=0 ; \\
& w_{1}+w_{1} \eta_{13} p c m_{1}+w_{2} \eta_{23} p c m_{2}+w_{3} \eta_{33} p c m_{3}=0,
\end{aligned}
$$

em que

$$
w_{i}=\frac{p_{i} q_{i}}{\sum_{j=1}^{n} p_{i} q_{i}}, \frac{\partial c}{\partial q_{i}}=m c_{i}, \frac{p_{i}-m c_{i}}{p_{i}}=p c m_{i}, i=1,2,3 .
$$

O sistema (9) permite soluções para os três pcms. As elasticidades-preço totalmente colusivas são dadas por:

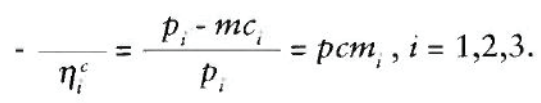

\section{APLICAÇÕES}

A principal aplicação de modelos que estimam demandas em nível de marcas comerciais tem sido o estudo do impacto das fusões de empresas nos preços pós fusão. O interesse nesses estudos decorre das exigências da lei antitruste dos EUA, a qual tem enfatizado mais os efeitos das fusões sobre o bem-estar social do que a concentração do mercado. Como os preços pós-fusão tendem a ser maiores quanto mais competidoras diretas forem as firmas envolvidas no processo de fusão, as elasticidades da demanda das marcas das firmas que pretendem se fundir são, geralmente, estimadas para se conhecerem as relações de concorrência entre essas marcas.

Nesse sentido, os trabalhos de Baker \& Breshnahan objetivaram, justamente, propor modelos para análise do efeito das fusões. Já Cotterill \& Haller desenvolveram um modelo como parte do testemunho que o prof. Cotterill deu, como expert, no processo que o advogado geral do Estado de Nova York moveu contra a aquisição do setor de cereais de café da manhã da Nbisco pela Kraft General Foods Inc. (produtora da linha de cereais Post).

Ainda no tocante a políticas de regulação, estimações de modelos semelhantes 
ao de CFM dariam aos órgãos de regulação uma imagem mais clara sobre a existência e sobre o tipo de poder de mercado exercido em dada indústria. Esses seriam elementos diretamente relacionados com o uso de poder de mercado, e não com o poder de mercado potencial, que pode ser inferido do grau de concentração do mercado. Os resultados das estimações mostrariam também se as estratégias das firmas mudaram em decorrência de alterações na política de regulação. Um caso em que esse tipo de análise se aplica é o da indústria americana de cereais de café da manhã. Até 1981, esta indústria, que se concentra quase que totalmente nas mãos de quatro empresas, havia sido bastante vigiada pelos órgãos de implementação da lei antitruste dos EUA. Após 1981, com as medidas adotadas pelo governo Reagan, o setor passou a funcionar livre da investigação federal (Preston \& Connor, 1993). Uma hipótese que pode ser testada pelo modelo é se as empresas passaram a trabalhar mais cooperativamente após a flexibilização das investigações federais.

Outra possibilidade de uso das estimações das elasticidades da demanda das marcas, ainda pouco explorada, é no setor de tomada de decisão empresarial. O cálculo de índices, como os propostos por CFM, permite inferir o grau de sucesso de uma estratégia de diferenciação de produto e explicita também o comportamento adotado por marcas rivais. A título de exemplo, considere o processo de decisão sobre a entrada num mercado ou num segmento de mercado. Nesse caso, o conhecimento do poder de mercado unilateral (índice R) desfrutado pelas firmas estabelecidas no mercado sugere o grau de dificuldade da entrada e mesmo a necessidade de despender grandes somas em promoção. Ainda dentro do mesmo exemplo, um mercado caracterizado por rivalidade (índice $\mathrm{CH}$ negativo) sugere ao ingressante potencial que as firmas estabelecidas provavelmente reagirão, vigorosamente, a qualquer perda de parcela de mercado.

\section{CONSIDERAÇÕES FINAIS}

O surgimento de dados desagregados em nível de marcas comerciais, oriundos de leitura de códigos de barras (scanners) nas máquinas registradoras de supermercados, tem sido um instrumento poderoso para o entendimento das estratégias concorrenciais das empresas num ambiente em que predomina a segmentação dos produtos em inúmeras marcas comerciais. Esses dados têm sido comercializados por empresas privadas, tais como IRI, Nielsen e SAMI, principalmente para outras empresas que querem acompanhar seus próprios desempenhos (e os de suas rivais) no mercado. No Brasil, com a difusão do uso de códigos de barras e com a chegada de firmas de levantamento de dados, como a Nielsen, dados dessa natureza estarão provavelmente disponíveis num futuro próximo.

Os modelos teóricos têm acompanhado a disponibilidade dos dados, e o modelo de CFM surge como uma alternativa altamente atraente para estudos dessa natureza, visto que ele é mais geral do que seus predecessores e permite a mensuração dos dois tipos de poder de mercado, separadamente. Problemas que ainda 
existem na estimação de modelos como este residem no grande número de marcas vigentes em muitos dos mercados, o que gera problemas de perdas de graus de liberdade que, em muitos casos, impedem a estimação do sistema completo. A principal alternativa usada tem sido os modelos de maximização de utilidade em estágios múltiplos, os quais, entretanto, não têm resolvido a questão da definição dos grupos e das marcas pertencentes a cada grupo. Uma alternativa é o uso de modelos de preços hedônicos (hedonic prices), para definir os grupos a partir dos atributos das diferentes marcas ${ }^{8}$, mas o teste da adequação dos grupos definidos requer a estimação do modelo completo ${ }^{9}$, o que traz de volta a questão dos graus de liberdade.

Apesar dos problemas de estimação ainda existentes, modelos como o de CFM serão, certamente, bastante utilizados nos próximos anos, uma vez que permitem o estudo do poder de mercado num contexto, cada vez mais comum, de multimarcas, com um grau de profundidade que os métodos tradicionais de estudo da organização industrial, a partir de dados de empresas, não permitem. Além disso, desenvolvimentos de métodos de estimação e de modelos teóricos já estão acontecendo.

\section{REFERÊNCIAS BIBLIOGRÁFICAS}

BAKER, Jonathan B. \& BRESHNAHAN, Timothy F. (1985) "The Gains from Merger or Collusion in Product-Differentiated Industries." Journal of Industrial Economics, 33 (June):427-443.

BAKER, Jonathan B. \& BRESHNAHAN, Timothy F. (1988) "Estimating the Residual Demand Curve Facing a Single Firm.” International Journal of Industrial Organization, 6:283-300.

BAUMOL, W. J. (1982) "Contestable Markets: an Uprising in the Theory of Industry Structure". The American Economic Review, 72(1):1-15.

COTTERILL, Ronald W.; FRANKLIN, Andrew \& MA, Li Y. (1996) Measuring Market Power Effects in Differentiated Product Industries: An Application to the Soft Drink Industry. Food Marketing Policy Center Research Report, \# 32 (April). Department of Agricultural and Resource Economics, University of Connecticut.

COTTERILL, Ronald W \& HALLER, Lawrence E. (1997) An Economic Analysis of the Demand for RTE Cereal: Product Market Definition and Unilateral Market Power Effects. Food Marketing Policy Center Research Report, \# 35 (October). Department of Agricultural and Resource Economics, University of Connecticut.

DEATON, A. \& MUELBAUER, J. (1980) “An Almost Ideal Demand System.” The American Economic Review, 70(June):312-26.

GOLDMAN, S.M. \& UZAWA, H. (1964) “A Note on Separability in Demand Analysis.” Econometrica, 32:387-398.

GREER, D.F. (1984) Industrial Organization and Public Policy. 2nd ed. New York: Macmillan.

HAUSMAN, J.; LEONARD, G. \& ZONA, J.D. (1994) “Competitive Analysis with Differentiated Products.” Annales D’Economie et de Statistique. (34):159-180.

\footnotetext{
${ }^{8}$ Ver Stanley \& Tschirhart (1991), para um exemplo de aplicação de preços hedônicos ao mercado de cereais de café da manhã.

${ }^{9}$ Ver Goldman \& Uzawa (1964).
} 
MA, Li-Yu. (1997) “An Econometric Analysis of Competition in a Differentiated Product Industry: The U.S. Read-to-Eat Cereal Industry.” Ph.D. Dissertation, University of Connecticut, 1997.

MARTIN, S. (1993) Industrial Economics. 2nd ed. New York: Macmillan.

PRESTON, Warren P. \& CONNOR, John M. (1993) “An Economic Investigation of Federal Antitrust Enforcement in the Food System.” In: Cotterill, Ronald W. (editor). Competitive Strategy Analysis in the Food System. Boulder: Westview Press, Inc.

STANLEY, Linda R. \& TSCHIRHART, John. (1991) "Hedonic Prices for a Nondurable Good: The Case of Breakfast Cereals." Review of Economics and Statistics, 73: 537-541. 